\title{
Analysis And Research On Somatosensory Controller
}

\author{
Liguo Zheng \\ Engineering Research Center \\ Jilin VIXO Animation, Comics \& Games Technology \\ Co.,Ltd. \\ Changchun, China \\ e-mail: 415380877@qq.com
}

\author{
Qingqing Wang \\ Engineering Research Center \\ Jilin VIXO Animation, Comics \& Games Technology \\ Co.,Ltd. \\ Changchun, China \\ e-mail: wangqingqing314@163.com
}

\begin{abstract}
There is a considerable gap between traditional input devices such as Keyboard, mouse, and touch panels and 3D controllers somatosensory. Somatosensory controller is developing rapidly in recent years. The user can control the device without any complicated, who can interact with the content immersive using of the hands, feet, arms and other body parts and even the eyes. Although somatosensory technology and equipment face with the difficulties of the cost of hardware and software environment which cannot be crossed. Both the Wii, Kinect and Leap Motion, which compare with the traditional mouse and keyboard and gamepad still cannot be able to afford for Public. So nature cannot be more attractive to attract users to buy and use. However, human-computer interaction to become the future trend cannot be ignored. To give the user a comprehensive and specific understanding about somatosensory controller, In this paper, analyze and research the performance and technical characteristics of several mainstream controller. Obtained advantages, disadvantages and scope of somatosensory controllers which come from different manufacturers. So there is a very good interactive experience by analyzing in this paper.
\end{abstract}

Keywords-3D somatosensory controller; iMotion; Wii; Kinect; Leap Motion

\section{INTRODUCTION}

The history of somatosensory game controller can be traced back to the beginning when the game was born. Light gun may be the advent of the first somatosensory game controller, its history almost as long as the home game with history: in June 1967, Ralph Baer and his assistant Harrison[1] together completed the world's first light gun - -it is also the Ralph • Bell designed the world's first home game. The prototype of light gun is very simple, but Harrison got the inspiration from a toy gun, he put parts needed to install a toy gun, the completion of the first of the light gun. And this time, even the first commercial video game are just a laboratory prototype of Bale.

The biggest advantage of somatosensory game controller is that it can simulate the existing equipment in reality, so players can get closer to the real game experience in the game. Traditional game controller in terms of the ability to restore the real experience is obviously insufficient, but the manufacturer did not give up and try to improve in this area, such as the vibration function of Dual Shock's series handle, its biggest role is to enhance the spot they feel - just so compared with the real design somatosensory game controller concerned on, it looked too pale.

With the 2009 E3 Expo, Sony and Microsoft announced the somatosensory operation equipment which is for the home game console. Three major hosts have their own somatosensory game controller, seems to imply that the game industry has started to enter a universal sense of body era. From the data published of official, the somatosensory game controller PS MOVE of Sony designed for the PS3 is similar to the Wii Remote in terms of appearance or function t. Sony has repeatedly stressed PS Move operation more accurate, more comfortable, However, this highlights of the game controllers still lack a truly ingenious. In contrast, Microsoft is tailored XBOX360 Kinect game controller, new ideas to make the limelight at the show. Unconsciously somatosensory game has occupied a place in the game field.

\section{MAINSTREAM SOMATOSENSORY CONTROLLER}

A. iMotion

iMotion is a revolutionary $3 \mathrm{D}$ controller, with tactile feedback. It can control the application on the computer mobile phone, flat by setting the relevant action to achieve a certain trigger condition to realize the somatosensory control.

iMotion has a extremely accurate three-dimensional space capture ability, which can recognize your body action perfectly.

iMotion is equal to construct a virtual space to the user's touch, the user can control the specific operation to the virtual object on the screen in the air. The iMotion related applications install on the user's device, you can control the computer and other smart devices instead of a mouse and keyboard. Users can operate devices directly in the air by iMotion.

It has three LED lights on the surface, which are used to detect $\mathrm{X}, \mathrm{Y}, \mathrm{Z}$ coordinates and elevation planar axis, rotation angle. iMotion has gyroscope and accelerometer which can detect any of your actions accurately[2].

Touch feedback technology issues to the operator information via Bluetooth. iMotion has five kinds of different intensity and duration of feedback. You can feel 
you are "touch" the object on the screen, increasing feelings of control.
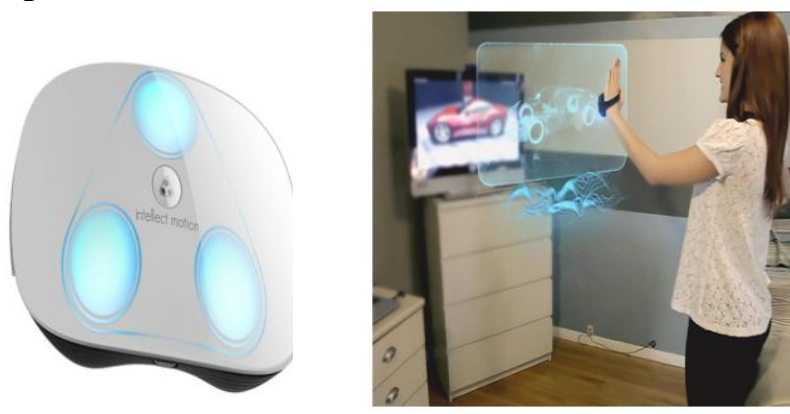

Figure1. iMotion and Application

$B$.

Wii

End of 2006, Nintendo released the Wii. Wii console can connect four handles via blue tooth technology. The wireless signal can be detected at a distance of 10 meters.[3] Wii remote and nunchuck handles are equipped with three-axis sensor. Wii Remote controller has a speaker, vibration function and expansion slots, and can be used as a locator in five meters from the screen

The design if the Wii use compact design, which can be placed horizontally and stacked vertically. Slot-drive of Wii can support single or double $12 \mathrm{~cm}$ Wii discs and $8 \mathrm{~cm}$ disc of NGC[4].

Users control the screen depending on the Wii, not yet completely done by hand. But the unprecedented use Wii controller, life information content unrelated the game and other features and services, opened a "revolution in mobile gaming".

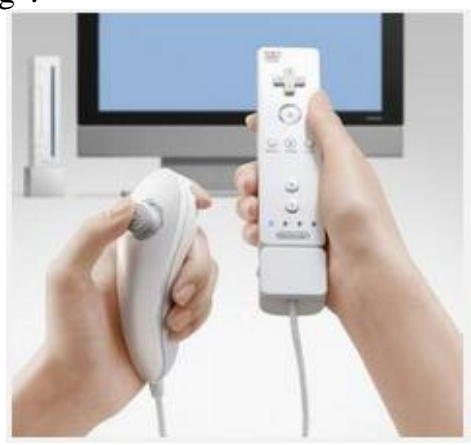

Figure2. The Exterior of Wii

\section{C.}

Kinect

Kinect is $\mathrm{XBOX} 360$ somatosensory peripheral peripherals which was announced formally by Microsoft at E3 June 2, 2009. Kinect revolutionized the single operation of the game, makes the idea of machine interaction more thoroughly unfolded. It is a 3D somatosensory camera, while it imported the instant motion capture, image recognition, microphone input, voice recognition, social interaction and other functions. Players can drive in the game and interact with other players, sharing pictures and information with other Xbox players via the Internet using this technology.

The emergence of Kinect evolved the natural user interaction to a new level. Kinect is equipped with a resolution of $640 \times 480$ of RGB VGA camera, a depth sensor and a multi-array microphone which can be identified the distance, action and even recognize and accept the voice commands of the players, and all this in real time[5].

Players need to make a variety of actions in front of the screen, the game character on the appropriate action to make the same. Even NPC chat using voice and make the world we live in and the screen on the other side of the world ever closer to the viewer are all marvel. Undoubtedly, Kinect can bring great fun for players of the game - the premise is that there is a large enough living room .......

With PrimeSense software and camera detection, Kinect capture user gestures, and then compare captured image with human model with own internal. Each human model with internal objects saved will be creating a model of skeletal-related system and then to convert the model into a virtual role that by identifying the key parts of the human skeleton model operates triggered. With the help of a virtual skeleton model, the system can identify 25 key parts of the human body. Based on this research add the new technology of recognition the human standing / sitting[6].

By depth of field camera and RGB camera, Kinect can put 3D images into the screen[7]. Simultaneously capture color images, infrared images, and the user's gesture, according to data given by Microsoft, and then convert these into sign language game control. Kinect can simultaneously track up to six players, but only two players can simultaneously operate a game.

Compared with the Wii, Kinect Another fascinating factor is that it does not need handle manipulation. Waved your arm can switch menu and control menu by voice, it all looks very sci-fi. Kinect allows players to somatosensory operate through a simple wave.

In the center of this Kinect players can control the menu by action, such as waving a hand from right to left can view the content. At the same time the hands do not move somewhere you can access the contents of their own interest.
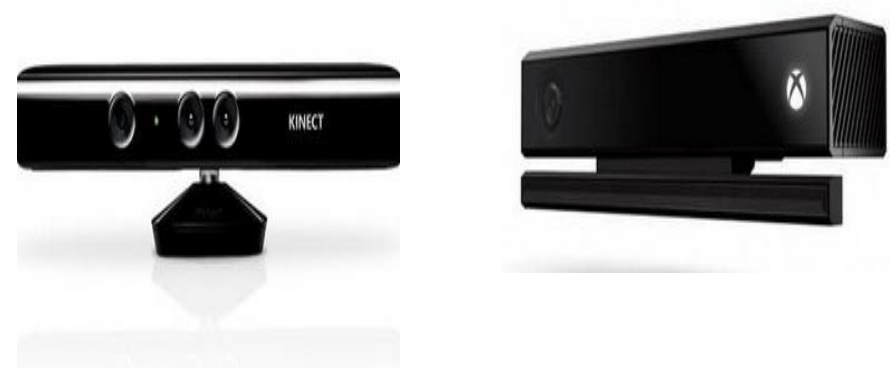

Figure3. Kinect1.0

Figure4. Kinect2.0

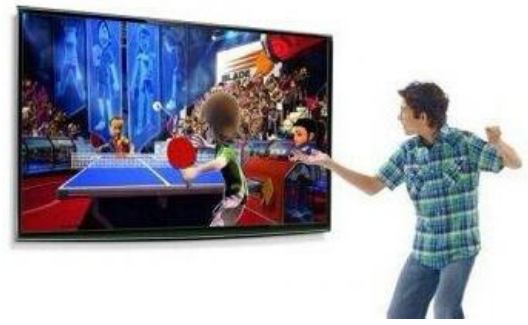

Figure5. Kinect Application 
Leap Motion is which is researched and produced for the PC and Mac somatosensory controller a new type of human-computer interaction devices. The function of Leap Motion is similar to Kinect. Users can control the $\mathrm{PC}$ and Mac computers via gestures.

But Leap Motion and Kinect works with completely different. Leap Motion uses Infrared and camera to replace the Kinect depth image, the coverage area is far away Microsoft somatosensory controller. But Leap Motion somatosensory performance is not comparable by all current depth cameras: it can simultaneously track 10 fingers of users, up to one hundredth of a millimeter accuracy, and only slightly lower than the refresh rate of the display[8][9].

Leap Motion provides motion capture of three axes, and now the use of these applications are only two axes, the data of "depth" has not used.[10] Can imagine that, if holographic display technology will be practical in the future, Leap Motion would be very good interfaces between real and virtual.

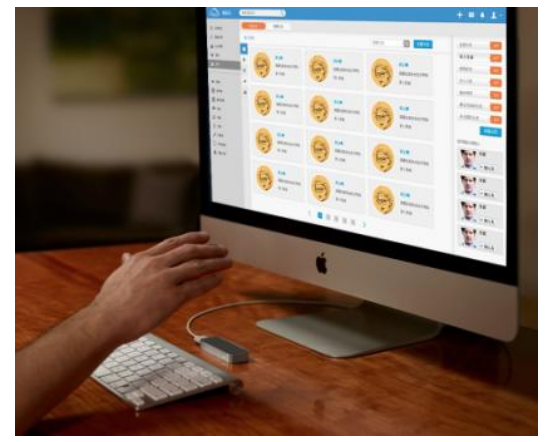

Figure6. Leap Motion Application

\section{E.}

\section{DUO}

Duo uses two PlayStation Eye cameras to detect user manipulation gesture. This camera is one of the important parts of Sony PlayStation3 game console. You can buy video game accessories in the store. From the official demo video, sensitivity and accuracy of Duo not only lose Leap Motion, the minimum detection range also seems much better than the latter from official demo video. Video also shows that users can not only use Duo for "Fruit Ninja" game, also the same as the Kinect compatible Windows8 gesture commands.

Duo's in public investment and financing platform Kickstarter website, fans pay only \$ 20 (about 124 yuan) to get its production instructions, including a detailed list of accessories as well as a CAD drawing. Since all parts are available in stores Duo available, so fans can refer to the drawings DIY directly assembled one of their own 3D somatosensory controller. Duo of sensitivity and accuracy not only lose Leap Motion, the minimum detection range also seems much better than the latter.
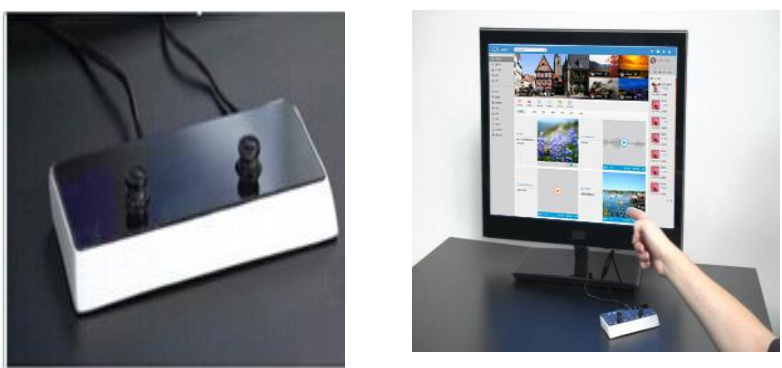

Figure7. DUO and Application

$$
F \text {. }
$$

CubeSense

CubeSense is a somatosensory control chip by the development of a new shoreline Co. CubeSense somatosensory controller chip base on the principles of stereoscopic vision. It capture three-dimensional images by two ordinary camera head, on this basis, identify the gestures and body movements to control smart devices.

CubeSense is the size of about $15 \mathrm{~cm}$ long, two knuckles wide. Plus the cover is reminiscent of Microsoft's Kinect sensor, but very different target markets. This device processing speed is 60fps/s. Only supports single-touch measurement, its accuracy can be reduced to a single finger size $(1 \mathrm{~mm})$, lens range is up to 120 degrees of wide-angle. While king deeply measurement allows the system to identify in front of people and dispense background interference.

One of the highlights of CubeSense Box is that get rid of traditional remote control. It use 3D gesture to achieve remote control of the TV interface. At the same time the internal configure a high-precision depth sensor chip CubeSenseTM. The chip independent designed by the new shoreline who has all the patents. Based on the principle of three-dimensional imaging of the human binocular vision, it can reality perception of human gestures in 3D space using only two ordinary cameras. Compared with other current depth of the chip, CubeSenseTM chip is more universal and economic feasibility. It supports a maximum resolution of $1280 *$ 960 original calculations.

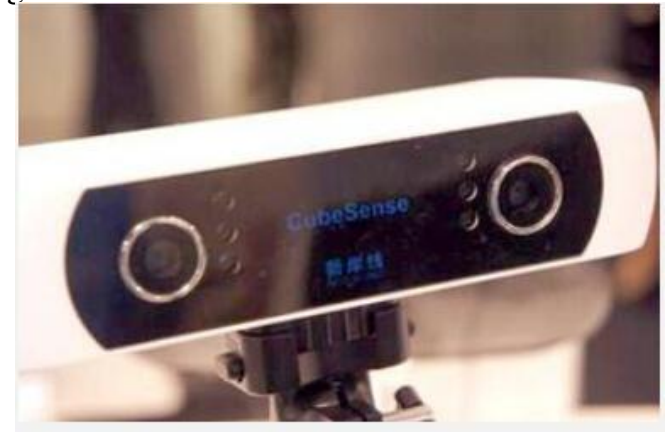

Figure8. CubeSense

\section{PERFORMANCE COMPARISION}

Through the above analysis, get the table as follows. 
TABLE I. INDEX CONTRAST OF SOMATOSENSORY CONTROLLERS

\begin{tabular}{|l|l|l|l|l|l|}
\hline & iMotion & Wii & Kinect & $\begin{array}{l}\text { Leap } \\
\text { Motion }\end{array}$ & DUO \\
\hline Company & $\begin{array}{l}\text { Intellect } \\
\text { Motion }\end{array}$ & Nintendo & $\begin{array}{l}\text { Micro- } \\
\text { soft }\end{array}$ & Leap & $\begin{array}{l}\text { Code } \\
\text { Labor- }\end{array}$ \\
\hline $\begin{array}{l}\text { Ranging } \\
\text { Element }\end{array}$ & $\begin{array}{l}\text { Gyro } \\
\text { scopes, } \\
\text { Accel- } \\
\text { ero- } \\
\text { meters }\end{array}$ & bluetooth & $\begin{array}{l}\text { RGB } \\
\text { VGA } \\
\text { camera }\end{array}$ & $\begin{array}{l}\text { Infrared } \\
\text { camera }\end{array}$ & $\begin{array}{l}\text { PS3 Eye } \\
\text { camera }\end{array}$ \\
\hline $\begin{array}{l}\text { Camera } \\
\text { Resolution }\end{array}$ & $\begin{array}{l}1280^{*} \\
800\end{array}$ & $\backslash$ & $\begin{array}{l}640^{*} \\
480\end{array}$ & $640^{*} 240$ & $\begin{array}{l}640^{*} \\
480\end{array}$ \\
\hline $\begin{array}{l}\text { Gesture } \\
\text { Recognition }\end{array}$ & $\sqrt{ }$ & $\times$ & $\sqrt{ }$ & $\sqrt{ }$ & $\sqrt{ }$ \\
\hline Price & $\$ 49$ & $\$ 150$ & $\$ 400$ & $\$ 69.99$ & $\$ 140$ \\
\hline
\end{tabular}

\section{SUMMARY}

This article describes the five kinds of somatosensory controller, which is a development process of somatosensory controller. From Nintendo's Wii to Microsoft's Kinect, somatosensory controller is not limited to games, which has gone from the control to handle the non-contact sensor feedback to touch and broken through the man-machine control limits. Domestic ASUS Xtion PRO LIVE and new shoreline's CubeSense are done better in somatosensory control. But compared with foreign products popularity is not enough, VIXO being developed GameBody which has speech recognition, gesture recognition, motion capture and other functions. Advancement of technology allow operation of electronic products with each passing day, from an external device to the finger skin, so the gap between the body and the device has been gradual elimination. We can say " the third revolution of Human-Computer Interaction" has ignited.

\section{ACKNOWLEDGMENT}

In the process of research work and writing papers, I get support and help from 2013 Jilin high-tech industry development funds "somatosensory interactive game development engine system integration industry"(No.2013G06). So I am very grateful for them.

\section{REFERENCES}

[1] Hatfield, Daemon, "GDC 2008: Ralph Baer Receiving Pioneer Award - The "father of videogames" is getting props," Retrieved $8 / 7 / 09$.

[2] http://www.ifanr.com/355298

[3] Chang Rong, "The Researeh and Implementation of the Key Multitoueh Technology Based on WiiRemote[D]," Kunming University of Science and Technology, 2011.

[4] Pei Y.C, May C.K, Jessy Lee, C.C.Jay Kuo, Wiistick: Enhancing Motion Recognition Capapility for Wii systems, Circuits and Systems, 2009, 1445-1448.

[5] Shi Manyin, "Research on the Technology and Working Principle of Kinect," Natural Science Journal of Harbin Normal University, vol.29, Mar. 2013, pp. 83-86.

[6] Erica Naone, "Microsoft Kinect: How the Device Can Respond to Your Voice and Gestures[Z]," Pioneering with Science and Technology, 2011;4(04);82-83.

[7] ZHANG Di, "Discussion of the Kinect Applications," Logistics Engineering and Management, vol.34, June 2012, pp. 3941,10.3969/j.issn.

[8] Jiang Yuchao, Somatosensory menacing--Leap motion is not the same [J]. Computer fans, 2013(11).

[9] Zoltan Tomori, Marian Antalik, Peter Kesa, Jan Kanka, Petr Jakl, Mojmir Sery, Silvie Bernatova, Pavel Zemanek, "Holographic Raman Tweezers Controlled by Hand Gestures and Voice Commands", The International Symposium on Photonics and Optoelectronics(SOPO 2013), IEEE Press, Mar. 2013, pp. 331-336, doi:10.4236/opj.2013.32B076.

[10] R. Bowman, D. Preece, G. Gibson and M. Padgett, "Stereoscopic Particle Tracking for 3D Touch, Vision and Closed-loop Control in Optical Tweezers," Journal of Optics, Vol. 13, No. 4, 2011, p.044003. doi:10.1088/2040-8978/13/4/044003 